\title{
AMOEBA BASIS OF ZERO-DIMENSIONAL VARIETIES
}

\author{
MOUNIR NISSE
}

\begin{abstract}
We show that the amoeba of a zero-dimensional variety consisting of a finite number of points has a finite basis. In other words, it is the intersection of finitely many hypersurface amoebas.
\end{abstract}

\section{INTRODUCTION}

Let $K$ be an algebraically closed field equipped with a non-trivial real valuation $\nu: K \rightarrow \mathbb{R} \cup\{\infty\}$. The tropical variety (or non-Archimedean amoeba) $\mathcal{T} \operatorname{rop}(\mathcal{I})$ of an ideal $\mathcal{I} \subset K\left[x_{1}, \ldots, x_{n}\right]$ is defined as the topological closure of the set

$$
\nu(V(\mathcal{I})):=\left\{\left(\nu\left(x_{1}\right), \ldots, \nu\left(x_{n}\right)\right) \mid\left(x_{1}, \ldots, x_{n}\right) \in V(\mathcal{I})\right\} \subset \mathbb{R}^{n},
$$

where $V(\mathcal{I})$ denotes the zero set of $\mathcal{I}$ in $\left(K^{*}\right)^{n}$ (see for example [MS-09]). A tropical basis for $\mathcal{I}$ is a generating set $\mathscr{B}=\left\{g_{1}, \ldots, g_{l}\right\}$ of $\mathcal{I}$ such that

$$
\mathcal{T} \operatorname{rop}(\mathcal{I})=\bigcap_{j=1}^{l} \mathcal{T} \operatorname{rop}\left(\mathcal{I}_{g_{j}}\right),
$$

where $\mathcal{I}_{g_{j}}$ denotes the principal ideal generated by the polynomial $g_{j}$. Bogart, Jensen, Speyer, Sturmfels and Thomas [BJSST-07] gave a lower bound on the size of such bases when $K$ is the field of Puiseux series $\mathbb{C}((t))$ and the ideal $\mathcal{I}$ is linear with constant coefficients. In [HT-09], Hept and Theobald showed that any tropical variety has a finite tropical basis. The archimedean amoeba $\mathscr{A}(V)$ of a subvariety $V$ of the complex torus $\left(\mathbb{C}^{*}\right)^{n}$ is its image under the coordinatewise logarithm map. Archimedean amoebas were introduced by Gelfand, Kapranov and Zelevinsky in 1994 GKZ-94]. The coamoeba of a subvariety of $\left(\mathbb{C}^{*}\right)^{n}$ is its image under the coordinatewise argument map to the real torus $\left(S^{1}\right)^{n}$ (see e.g., [NS-12] for more details about coamoebas). The purpose of this note is to show that Hept and Theobald's theorem has an analogue for archimedean amoebas of codimension $n$.

1991 Mathematics Subject Classification. 14T05, 32A60.

Key words and phrases. Amoebas, hyperplane amoebas, and amoeba basis.

This research is partially supported by NSF MPS grant DMS-1001615, and Korea Institute for Advanced Study (KIAS), Seoul, South Korea. 
Let $V_{f}$ denotes the hypersurface with defining polynomial $f$. If the variety $V$ is generated by the set of polynomials $\left\{g_{i}\right\}_{i=1}^{l}$ with the following properties:

(i) $\mathscr{A}(V)=\bigcap_{i=1}^{l} \mathscr{A}\left(V_{g_{i}}\right)$;

(ii) $\mathscr{A}(V) \subsetneq \bigcap_{i \in\{1, \ldots, l\} \backslash s} \mathscr{A}\left(V_{g_{i}}\right)$ for every $1 \leq s \leq l$,

then we say that $\left\{g_{i}\right\}_{i=1}^{l}$ is a finite amoeba basis of $\mathscr{A}(V)$.

It was shown in $[\mathrm{N}-14$ that the amoeba of a generic complex algebraic variety of codimension $1<r<n$ does not have a finite basis. In other words, it is not the intersection of finitely many hypersurface amoebas. The aim of this paper is to show that if the codimension of our variety $V$ is equal to $n$ (i.e., $V$ is a finite number of points), then its amoeba has a finite basis. In other words, the main result of Hept and Theobald in [HT-09] does have an analogue for archimedean amoebas of 0 -dimensional complex algebraic varieties.

Theorem 1.1. Let $V \subset\left(\mathbb{C}^{*}\right)^{n}$ be a zero-dimensional variety consisting of a finite number of points. Then the amoeba $\mathscr{A}(V)$ of $V$ has an amoeba basis.

\section{Preliminaries}

Let us recall the description of hyperplane amoebas given by Forsberg, Passare and Tsikh [FPT-00] and some of their feature that we will use in our proof. In Corollary 4.3 [FPT-00], Forsberg, Passare and Tsikh showed the following:

Proposition 2.1 (Forsberg-Passare-Tsikh). The amoeba $\mathscr{A}\left(V_{f}\right)$ of an affine complex function $f(z)=b_{0}+b_{1} z_{1}+\cdots+b_{n} z_{n}$ is equal to the closed (possibly empty) subset in $\mathbb{R}^{n}$ defined by the inequalities

$$
\begin{aligned}
\log \left|b_{0}\right| & \leq \log \left(\sum_{k=1}^{n}\left|b_{k}\right| e^{u_{k}}\right), \\
u_{j}+\log \left|b_{j}\right| & \leq \log \left(\left|b_{0}\right|+\sum_{k \neq j}^{n}\left|b_{k}\right| e^{u_{k}}\right),
\end{aligned}
$$

for $j=1, \ldots, n$, and where the variables are the $u_{i}$ 's.

By setting $r_{k}=e^{u_{k}}$ for $k=1, \ldots, n$, Proposition 2.1 says that the image by the exponential map of the amoeba of an affine complex hyperplane defined by an affine linear complex function $f(z)=b_{0}+$ $b_{1} z_{1}+\cdots+b_{n} z_{n}$ is equal to the set of points $\left(r_{1}, \ldots, r_{n}\right) \in \mathbb{R}_{+}^{n}$ that 
satisfy the generalized triangle inequalities given by:

$$
\begin{aligned}
\left|b_{0}\right| & \leq \sum_{k=1}^{n}\left|b_{k}\right| r_{k}, \\
r_{j}\left|b_{j}\right| & \leq\left|b_{0}\right|+\sum_{k \neq j}^{n}\left|b_{k}\right| r_{k}, \quad \text { for } j=1, \ldots n .
\end{aligned}
$$

Let $\mathscr{P}$ be an affine linear subspace of codimension greater than one in $\left(\mathbb{C}^{*}\right)^{n}$. Then the amoeba of $\mathscr{P}$ is much smaller than the intersection of all amoebas of hyperplanes containing $\mathscr{P}$ (i.e., the amoeba of $\mathscr{P}$ is strictly contained in the intersection of all amoebas of hyperplanes containing $\mathscr{P})$. For example, let $L$ be the affine line in $\left(\mathbb{C}^{*}\right)^{3}$ parametrized as follows:

$$
\begin{aligned}
\rho: \mathbb{C} \backslash\{0,-u, v\} & \longrightarrow\left(\mathbb{C}^{*}\right)^{3} \\
z & \longmapsto(z, z+u,-z+v),
\end{aligned}
$$

where $u$ and $v$ are strictly positive numbers, and denote by $\mathscr{A}(L)$ the amoeba of $L$. Let $x$ be the point in $\mathbb{R}^{3}$ with coordinate $(1, u, v)$. It is clear that if $|z|=1$ and $|z+u|=u$, then $|-z+v| \neq v$ for all $v \in \mathbb{R}_{+}$. Indeed, $|z|=1$ and $|z+u|=u$ implies that $\arg (z) \in\left(\frac{\pi}{2}, \frac{3 \pi}{2}\right)$, and then $\arg (-z) \in\left(\frac{-\pi}{2}, \frac{\pi}{2}\right)$. This means that $|-z+v| \neq v$ for all strictly positive number $v$. In other words, the point $(0, \log u, \log v) \notin \mathscr{A}(L)$. Now assume that $|u|>1$, and $|v|>1$. We claim in this case that the point $(0, \log u, \log v)$ is contained in all amoebas of hyperplanes containing $L$. In fact, let $\mathscr{H}_{f}$ be a hyperplane in $\left(\mathbb{C}^{*}\right)^{3}$ containing $L$ and with defining polynomial $f\left(z_{1}, z_{2}, z_{3}\right)=a_{0}+a_{1} z_{1}+a_{2} z_{2}+a_{3} z_{3}$. Since $(0, u, v) \in L$ and $(1,1+u,-1+v) \in L$, the coefficients must satisfy following relations:

$$
a_{0}=-\left(u a_{2}+v a_{3}\right) \text { and } a_{1}=a_{3}-a_{2} .
$$

In other words, $f\left(z_{1}, z_{2}, z_{3}\right)=-\left(u a_{2}+v a_{3}\right)+\left(a_{3}-a_{2}\right) z_{1}+a_{2} z_{2}+a_{3} z_{3}$. Moreover, since by assumption $|u|>1$, and $|v|>1$, then for all complex numbers $a_{2}$ and $a_{3}$, we have the following inequalities:

$$
\left\{\begin{aligned}
\left|u a_{2}+v a_{3}\right| & \leq\left|a_{3}-a_{2}\right|+u\left|a_{2}\right|+v\left|a_{3}\right|, \\
u\left|a_{2}\right| & \leq\left|u a_{2}+v a_{3}\right|+v\left|a_{3}\right|+\left|a_{3}-a_{2}\right|, \\
v\left|a_{3}\right| & \leq\left|u a_{2}+v a_{3}\right|+u\left|a_{2}\right|+\left|a_{3}-a_{2}\right|, \\
\left|a_{3}-a_{2}\right| & \leq\left|u a_{2}+v a_{3}\right|+u\left|a_{2}\right|+v\left|a_{3}\right|
\end{aligned}\right.
$$

where the three first inequalities comes from the triangular inequalities of the triangle with vertices $0, u a_{2}$, and $u a_{2}+v a_{3}$. The last inequality comes from the assumption on $u$ and $v$ and the triangular inequalities of the triangle with vertices $0, a_{3}$, and $a_{3}-a_{2}$.

By Proposition 2.1, the point $(0, \log u, \log v)$ is contained in any amoeba of hyperplane containing $L$. This example shows that for lines (or even a planes of dimension $k$ ) in $\left(\mathbb{C}^{*}\right)^{n}$, the degree of functions in its amoeba basis must contain functions of degree $d>1$. Moreover, it was shown in 
N-14 that the amoeba in this case does not have a basis (i.e., it cannot be the intersection of a finite number of hyperplane amoebas). In this paper, we show that amoeba basis exists in dimension zero (which is not obvious to see directly).

\section{Proof OF THE MAIN RESUlt}

Let $V:=\left\{v^{(1)}, v^{(2)}, \ldots, v^{(l)}\right\} \subset\left(\mathbb{C}^{*}\right)^{n}$ be a zero-dimensional variety consisting of $l$ different points. Let $\mathscr{A}(V):=\left\{w^{(1)}, w^{(2)}, \ldots, w^{(l)}\right\}$ be the amoeba of $V$. If $v^{(i)}$ is equal to $\left(v_{1}^{(i)}, \ldots, v_{n}^{(i)}\right) \in\left(\mathbb{C}^{*}\right)^{n}$, then we denote by $\left\|v^{(i)}\right\|_{0}$ the following sum:

$$
\left\|v^{(i)}\right\|_{0}=\sum_{k=1}^{n}\left|v_{k}^{(i)}\right|
$$

Using the construction of a system of $(n+1)$ polynomial equations given by Schroeter and de Wolff in [SW-13, let us consider the following system of $(n+1)$ polynomial equations in $n$ variables $z=\left(z_{1}, \ldots, z_{n}\right)$ which we denote by $(\mathscr{G})$ :

$$
\begin{aligned}
& g_{0}(z)=\prod_{i=1}^{l}\left(1-\frac{1}{\left\|v^{(i)}\right\|_{0}} \sum_{k=1}^{n} \frac{\left|v_{k}^{(i)}\right|}{v_{k}^{(i)}} z_{k}\right)=0, \\
& g_{j}(z)=\prod_{i=1}^{l}\left(1-\frac{1+|| v^{(i)} \|_{0}-\left|v_{j}^{(i)}\right|}{v_{j}^{(i)}} z_{j}+\sum_{k \in\{1, \ldots, n\} \backslash j} \frac{\left|v_{k}^{(i)}\right|}{v_{k}^{(i)}} z_{k}\right)=0,
\end{aligned}
$$

for $j=1, \ldots n$. We can check that the amoeba of each polynomial $g_{j}$ for $j=0,1, \ldots, n$ is an arrangement of $l$ hyperplane amoebas (counted with multiplicity, because it can happen that $v^{(i)} \neq v^{(m)}$ but $\left\|v^{(i)}\right\|_{0}=$ $\left\|v^{(m)}\right\|_{0}$ or $v_{j}^{(i)}=v_{j}^{(m)}$ for some $\left.j\right)$. For $i=1, \ldots, l$, we denote by $\mathscr{H}_{j}^{(i)}$ the hyperplane with defining polynomial $g_{j}^{(i)}$ which is the $i^{\text {th }}$ factor of $g_{j}$ for $j=0,1, \ldots, n$, i.e.,

$$
g_{0}^{(i)}(z)=1-\frac{1}{\left\|v^{(i)}\right\|_{0}} \sum_{k=1}^{n} \frac{\left|v_{k}^{(i)}\right|}{v_{k}^{(i)}} z_{k}, \quad \text { for } i=1, \ldots, l
$$

and

$$
g_{j}^{(i)}(z)=1-\frac{1+|| v^{(i)}||_{0}-\left|v_{j}^{(i)}\right|}{v_{j}^{(i)}} z_{j}+\sum_{k \in\{1, \ldots, n\} \backslash j} \frac{\left|v_{k}^{(i)}\right|}{v_{k}^{(i)}} z_{k},
$$

for $i=1, \ldots, l$ and $j=1, \ldots, n$.

By construction, each $v^{(i)}$ is a solution of the system $(\mathscr{G})$, and then $\mathscr{A}(V) \subset \bigcap_{j=0}^{n} \mathscr{A}\left(V_{g_{j}}\right)$, where $\mathscr{A}\left(V_{g_{j}}\right)$ denotes the arrangement of hypersurface amoebas defined by the polynomial $g_{j}$. We can rewrite the 
system $(\mathscr{G})$ as follows

$$
\begin{array}{cccccccc}
g_{0}(z) & = & g_{0}^{(1)}(z) & g_{0}^{(2)}(z) & \ldots & g_{0}^{(l)}(z) & = & 0 \\
g_{1}(z) & = & g_{1}^{(1)}(z) & g_{1}^{(2)}(z) & \ldots & g_{1}^{(l)(z)} & = & 0 \\
\vdots & \vdots & \vdots & \vdots & \vdots & \vdots & \vdots & \vdots \\
g_{n}(z) & = & g_{n}^{(1)}(z) & g_{n}^{(2)}(z) & \ldots & g_{n}^{(l)}(z) & = & 0
\end{array}
$$

Let $w \in \bigcap_{j=0}^{n} \mathscr{A}\left(V_{g_{j}}\right)$ and $u \in\left(\mathbb{C}^{*}\right)^{n}$ such that $\log (u)=w$. As we know, the amoeba defined by $g_{j}$ is the union of the hyperplane amoebas $\mathscr{A}\left(\mathscr{H}_{j}^{(i)}\right)$ for $i=1, \ldots, l$, then for any $j \in\{0, \ldots, n\}$ there exists $i \in\{1, \ldots, l\}$ such that $w \in \mathscr{A}\left(\mathscr{H}_{j}^{(i)}\right)$.

Lemma 3.1. Let $w$ be a point in the complement of the amoeba $\mathscr{A}(V)$. Then for any $i \in\{1, \ldots, l\}$ there exists a nonempty subset $S_{i}(w) \subset$ $\{0,1, \ldots, n\}$ such that $w \notin \mathscr{A}\left(V_{g_{j}^{(i)}}\right)$ for all $j \in S_{i}(w)$.

Proof. Let $w$ be a point in the complement of the amoeba $\mathscr{A}(V)$. Then $w \neq w^{(i)}$ for all $i=1, \ldots, l$. Let $u \in\left(\mathbb{C}^{*}\right)^{n}$ with $\log (u)=w$ and assume $\log (u) \in \bigcap_{j=0}^{n} \mathscr{A}\left(g_{j}^{(i)}\right)$. This implies that $\|u\|_{0}<\left\|v^{(i)}\right\|_{0}$ or there exists $j \in\{1, \ldots, n\}$ such that $\left|u_{j}\right|>\left|v_{j}^{(i)}\right|$. In other words, $1>\|u\|_{0}=\sum_{k=1}^{n}\left|u_{k}\right|$ or there exists $j \in\{1, \ldots, n\}$ such that:

$$
\left|u_{j}\right|>1+\sum_{k \in\{1, \ldots, n\} \backslash\{j\}}\left|u_{k}\right| .
$$

By Forsberg-Passare-Tsikh Proposition 2.1, this implies that there exists $j \in\{0,1, \ldots, n\}$ such that $\log (u)$ is contained in the complement component of the amoeba of $g_{j}^{(i)}$ with order given by the standard vector $e_{j}$. This is in contradiction with the fact that $\log (u) \in \bigcap_{j=0}^{n} \mathscr{A}\left(g_{j}^{(i)}\right)$ (see [SW-13] for more details).

Let us denote by $L_{i(1), \ldots, i(l)}:=L_{i(1)} \times L_{i(2)} \times \cdots \times L_{i(l)} \subset\{0,1, \ldots, n\}^{l}$, where $L_{i(j)}$ runs through all subsets of $\{0,1, \ldots, n\}$. Let $\mathscr{A}_{i(1), \ldots, i(l)}^{c}(V)$ be the subset of the complement $\mathscr{A}^{c}(V):=\mathbb{R}^{n} \backslash \mathscr{A}(V)$ of the amoeba defined as follows:

$\mathscr{A}_{i(1), \ldots, i(l)}^{c}(V):=\left\{w \in \mathscr{A}^{c}(V) \mid\left(S_{1}(w), S_{2}(w), \ldots, S_{l}(w)\right)=L_{i(1), \ldots, i(l)}\right\}$.

As we took all the possible subsets $L_{i(j)} \subset\{0,1, \ldots, n\}$, we can check that the amoeba complement $\mathscr{A}^{c}(V)$ is equal to the union of all the $\mathscr{A}_{i(1), \ldots, i(l)}^{c}(V)^{\prime}$ 's, i.e.,

$$
\mathscr{A}^{c}(V)=\bigcup_{(i(1), \ldots, i(l))} \mathscr{A}_{i(1), \ldots, i(l)}^{c}(V) .
$$


Lemma 3.2. With the above notation, there exist a finite number of polynomials $\left\{h_{s}\right\}_{s=1}^{r}$ of degree l such that:

(i) The set of solutions of the system $\left\{g_{j}(z)=h_{s}(z)=0\right\}$ for $j=0,1, \ldots, n$ and $s=1, \ldots, r$ is equal to $V$;

(ii) The intersection

$$
\left\{\bigcap_{j=0}^{n} \mathscr{A}\left(V_{g_{j}}\right)\right\} \bigcap\left\{\bigcap_{s=1}^{r} \mathscr{A}\left(V_{h_{s}}\right)\right\}_{s=1}^{r}
$$

is precisely $\mathscr{A}(V)=\left\{w^{(1)}, \ldots, w^{(l)}\right\}$.

Proof. Let $w \in \bigcap_{j=0}^{n} \mathscr{A}\left(V_{g_{j}}\right)$, then for any $j \in\{0,1, \ldots, n\}$ there exists $i(j) \in\{1, \ldots, l\}$ with $w \in \mathscr{A}\left(V_{g_{j}^{(i(j))}}\right)$, i.e., $w \in \bigcap_{j=0}^{n} \mathscr{A}\left(V_{g_{j}^{(i(j))}}\right)$. On the other hand, suppose that $w$ is contained in the complement of the amoeba $\mathscr{A}(V)$. Hence, by Lemma 3.1, and the construction above, there exists an index tuple $(i(1), \ldots, i(l))$ such that $w \in \mathscr{A}_{i(1), \ldots, i(l)}^{c}(V)$. Consider the set of all arrangement of $l$ hyperplane amoebas where each of these arrangements is defined by the product of the first order polynomials as follows:

$$
h_{j(1), \ldots, j(l)}(z):=g_{j(1)}^{(1)}(z) g_{j(2)}^{(2)}(z) \ldots g_{j(l)}^{(l)}(z),
$$

where $(j(1), \ldots, j(l)) \in L_{i(1), \ldots, i(l)}$. By construction, $V \subset V_{h_{j(1), \ldots, j(l)}}$, and $w \notin \mathscr{A}\left(V_{h_{j(1), \ldots, j(l)}}\right)$ for all index tuples $(j(1), \ldots, j(l)) \in L_{i(1), \ldots, i(l)}$. Let $\mathscr{B}$ be the subset of $\mathbb{R}^{n}$ defined as follows

$$
\mathscr{B}:=\left\{\bigcap_{j=0}^{n} \mathscr{A}\left(V_{g_{j}}\right)\right\} \bigcap\left\{\bigcap_{j(1), \ldots, j(l)} \mathscr{A}\left(V_{h_{j(1), \ldots, j(l)}}\right)\right\},
$$

where the intersection $\bigcap_{j(1), \ldots, j(l)} \mathscr{A}\left(V_{h_{j(1), \ldots, j(l)}}\right)$ is taken over all the index tuples in the $L_{i(1), \ldots, i(l)}$ 's. We claim that $\mathscr{B}$ is precisely the amoeba of $V$. Indeed, let $w \in \mathscr{B}$ and assume $w \notin \mathscr{A}(V)$. Then for each $i \in$ $\{1, \ldots, l\}$, there exists a nonempty subset $S_{i}(w) \subset\{0,1, \ldots, n\}$ such that $w \notin \mathscr{A}\left(V_{g_{j(i)}^{(i)}}\right)$ for all $j(i) \in S_{i}(w)$. Otherwise, using Proposition 2.1 and Lemma 3.1, the point $w$ will be equal to one of the $w^{(i)}$ 's. Namely, there exists an index tuple $(j(1), \ldots, j(l))$ such that $w$ is not in the amoeba of $V_{h_{j(1), \ldots, j(l)}}$ (because in the intersection $\mathscr{B}$, we took all possible arrangements). This is in contradiction with the fact that $w \in \mathscr{B}$. The other inclusion is given by construction. It remains to show that $V$ is equal to the set of solutions $V^{\prime}$ of the system $\left(\mathscr{S}^{\prime}\right)$ defined by

$$
\left\{g_{j}(z)=0\right\}_{j=0}^{n} \bigcap\left\{h_{j(1), \ldots, j(l)}(z)=0\right\}_{(j(1), \ldots, j(l))} .
$$

By construction, we have the first inclusion $V \subset V^{\prime}$. Lemma 3.3 implies that if $v$ is a solution of the system $\left(\mathscr{S}^{\prime}\right)$ then $v$ must be one of the $v^{(i)}$ 's, because by construction, the only solution of the system $\left(\mathscr{G}^{(i)}\right):=$ 
$\left\{g_{j}^{(i)}(z)=0 \mid j=0,1, \ldots, n\right\}$ is the point $v^{(i)}$. Hence, the number of solutions $\nu$ (counted with multiplicity) of the system $\left(\mathscr{S}^{\prime}\right)$ cannot exceed $l$. Since we know that $V \subset V^{\prime}$, then $\nu$ is also at least equal to $l$, and then $V^{\prime}$ should be equal to $V$.

Lemma 3.3. Assume $l \geq 2$ and $v \in\left(\mathbb{C}^{*}\right)^{n}$ be a solution of both systems $(\mathscr{G})$ and $(\mathscr{H})$ where the system $(\mathscr{H})$ consists of all equations $h_{j(1), \ldots, j(l)}(z)=0$, i.e.,

$$
(\mathscr{H}):=\left\{h_{j(1), \ldots, j(l)}(z)=0\right\}_{(j(1), \ldots, j(l))} .
$$

Then $v$ satisfies at least one system $\left(\mathscr{G}^{(i)}\right)$ for some $i \in\{1, \ldots, l\}$ with $\left(\mathscr{G}^{(i)}\right):=\left\{g_{j}^{(i)}(z)=0 \mid j=0,1, \ldots, n\right\}$.

Proof. Assume $v \notin V:=\left\{v^{(1)}, v^{(2)}, \ldots, v^{(l)}\right\}$, and for $i=1,2, \ldots, l$, let us denote by $R_{i}(v)$ the set of indices $j(i) \in\{0,1, \ldots, n\}$ such that $g_{j(i)}^{(i)}(v) \neq 0$. The index tuples $(j(1), j(2), \ldots, j(l))$ are contained in some set $L_{j(1), j(2), \ldots, j(l)}$. Recall that the system $(\mathscr{H})$ contains all the equations $h_{j(1), j(2), \ldots, j(l)}(z)=0$ with $(j(1), j(2), \ldots, j(l)) \in R_{i}(v)$. Assume that $v$ satisfies $g_{u(i)}^{(i)}(v)=0$ where the number of indices $u(i)$ with this property is equal to $s(i)$. Moreover, suppose that for each $i$ the number $s(i)$ is the maximum for this property. As the index tuples in the system $(\mathscr{H})$ contains the complement index tuples of the $(u(1), u(2), \ldots, u(l))$ 's and $v$ satisfies also the system $(\mathscr{H})$, then for some $i$, we can increase at least one of the numbers $s(i)$. This is in contradiction with the fact that these numbers are the maximum for the vanishing property. This means that there is an index $i \in\{1,2, \ldots, l\}$ with $g_{j}^{(i)}(v)=0$ for all $j \in\{0,1, \ldots, n\}$.

Proof of Theorem 1.1. Theorem 1.1 is an immediate consequence of Lemma 3.2 .

Example. Let $V:=\left\{v^{(1)}, v^{(2)}\right\} \subset\left(\mathbb{C}^{*}\right)^{2}$ be a set of two different points (i.e., $l=2)$. Assume that $\left\|v^{(1)}\right\|_{0} \neq\left\|v^{(2)}\right\|_{0}$. Consider the system ( $\left.\mathscr{G}\right)$ defined as follows:

$$
\begin{aligned}
& g_{0}(z)=g_{0}^{(1)}(z) \quad g_{0}^{(2)}(z)=0, \\
& g_{1}(z)=g_{1}^{(1)}(z) \quad g_{1}^{(2)}(z)=0 \\
& g_{2}(z)=g_{2}^{(1)}(z) \quad g_{2}^{(2)}(z)=0 .
\end{aligned}
$$

Where $g_{j}^{(i)}$ for $j=0,1,2$ and $i=1,2$ are defined as before. We add for $(\mathscr{G})$ the following polynomial equations: $g_{j}^{(i)} g_{r}^{(s)}$ with $j \neq r$ and $i \neq s$. We can check that the system

$$
\mathscr{B}=\left\{g_{j}\right\}_{j=0}^{2} \bigcup\left\{g_{j}^{(i)} g_{r}^{(s)}\right\}_{j, r=0,1,2 \text { with } i, s=1,2, j \neq r \text { and } i \neq s}
$$

is an amoeba basis of $\mathscr{A}(V)$ of length 9 and each of its element is of degree 2 . 


\section{REFERENCES}

[BJSST-07] T. Bogart, A.N. Jensen, D. Speyer, B. Sturmfels, and R.R. Thomas, Computing tropical varieties, J. Symb. Comp. 42, (2007), no. 1-2, $54-73$.

[FPT-00] M. Forsberg, M. PAssare And A. Tsikh, Laurent determinants and arrangements of hyperplane amoebas, Advances in Math. 151, (2000), 45-70.

[GKZ-94] I. M. Gelfand, M. M. Kapranov and A. V. Zelevinski, Discriminants, resultants and multidimensional determinants, Birkhäuser Boston 1994.

[HT-09] K. Hept And T. Theobald, Tropical bases by regular projections. Proc. Amer. Math. Soc., 137(7), 2233-2241, 2009.

[MS-09] D. Maclagan And B. Sturmfels, Introduction to Tropical Geometry. Graduate Studies in Math., Vol 161, American Math. Soc. (2015).

[N-14] M. Nisse, Amoeba basis does not exist in general, Preprint, arXiv: 1403.3912

[NS-12] M. Nisse And F. Sottile, The phase limit set of a variety, Algebra \& Number Theory, 7, (2013), 339-352.

[SW-13] F. Schroeter And T. DE WolfF, The boundary of amoebas, preprint, arXiv: 1310.7363 .

School of Mathematics KiAS, 87 Hoegiro Dongdaemun-gu, Seoul 130-722, South Korea.

E-mail address: mounir.nisse@gmail.com 